\title{
䋨irestre
}

\section{Criação de um aplicativo de estudo para residência médica baseado na perspectiva do usuário}

\author{
CREATION OF A MEDICAL RESIDENCY STUDY APPLICATION BASED ON THE USER'S \\ PERSPECTIVE
}

Gabriel Felipe Moreira de Souza', Erica Yamashita de Oliveira², Nathalia Ingrid Crosewski ${ }^{3}$, Gislaine Bonete ${ }^{4}$, Fernando Augusto Lavezzo Dias ${ }^{5}$

\author{
1 Graduando em medicina. Universidade Federal do \\ Paraná. \\ ORCID: https://orcid.org/0000-0001-9331-4727 \\ Email: gabrielflpsouza@gmail.com \\ 2 Médica. Universidade Federal do Paraná \\ ORCID: https://orcid.org/0000-0002-6938-7544 \\ Email: erica.y.oliveira@gmail.com \\ 3 Médica. Universidade Federal do Paraná. \\ ORCID: https://orcid.org/0000-0002-9568-2029 \\ Email: nathaliacrosewski@gmail.com \\ 4 Doutoranda em Fisiologia. Universidade Federal do \\ Paraná. \\ ORCID: https://orcid.org/0000-0003-4295-281 1 \\ Email: gislaine.fisiosm@gmail.com \\ 5 Doutora em Fisiologia. Universidade Federal do \\ Paraná. \\ ORCID: https://orcid.org/0000-0003-3951-2604 \\ Email: faldias@ufpr.br
}

Correspondência: Departamento de Fisiologia, Setor de Ciências Biológicas, Centro Politécnico,

Universidade Federal do Paraná - Avenida Coronel Francisco Heráclito dos Santos, 100, Jardim das Américas. Curitiba, PR - Brasil. CEP: 81531-980.

Copyright: Esta obra está licenciada com uma Licença Creative Commons Atribuição-NãoComercial 4.0 Internacional.

Conflito de interesses: os autores declaram que não há conflito de interesses.

\section{Como citar este artigo}

Souza GFM de; Oliveira EY de; Crosewski Nl; Bonete G; Dias FAL. Criação de um aplicativo de estudo para residência médica baseado na perspectiva do usuário. Revista de Saúde Digital e Tecnologias Educacionais. [online], volume 5, n. 2. Editor responsável: Luiz Roberto de Oliveira. Fortaleza, julho de 2020, p. 30-43. Disponível em: http://periodicos.ufc.br/resdite/index. Acesso em "dia/mês/ano".

Data de recebimento do artigo: $18 / 12 / 2019$

Data de aprovação do artigo: 25/05/2020

Data de publicação: 20/07/2020

\section{Resumo}

Introdução: Médicos recém-formados buscam o título de especialista, enfrentando processos seletivos concorridos. Cursos preparatórios e ferramentas digitais surgem diante da busca por aprovação. Assim, o objetivo do estudo foi a construção de um aplicativo focado em provas de residência médica baseado na perspectiva e opinião do usuário. Métodos: Foi realizado um estudo analítico transversal para investigar o entendimento e a perspectiva dos alunos sobre o processo de seleção e o uso de aplicativos, a partir desse estudo foi desenvolvido um aplicativo agregador de provas de residência médica. Resultados: A maioria dos alunos entrevistados (93,7\%) almejava fazer residência e entendia os custos envolvidos. Aplicativos para estudo eram frequentemente usados (72,7\%) pela maioria dos alunos $(86,8 \%$ ) e serviam para autoavaliação $(75,0 \%)$ e identificação de conteúdo deficitário (65,6\%). Foi possível desenvolver um aplicativo móvel que supriu parcialmente as necessidades relatadas 
pelos usuários. Conclusão: Aplicativos são ferramentas que auxiliam no estudo e na autoavaliação para seleção de residência médica, porém os disponíveis não suprem as necessidades do usuário. Foi desenvolvido o protótipo de um aplicativo móvel agregador de questões, adequado ao perfil deste usuário, que supre carências apontadas, como acesso grátis e possibilidade de criação de testes personalizados.

Palavras-Chave: Internato e Residência. Aplicativos Móveis. Educação Médica. Capacitação.

\section{Abstract \\ Introduction: Newly graduated doctors seek the title of specialist, facing competitive selection processes. Preparatory courses and digital tools appear in search of approval. Thus, the objective of the study was to build an application focused on medical residency tests based on the user's perspective and opinion. Methods: A cross- sectional analytical study was carried out to}

investigate students' understanding and perspective on the selection process and the use of applications. From this study, an application to aggregate medical residency evidence was developed. Results: Most of the interviewed students (93.7\%) wanted to do residency and understood the costs involved. Study apps were frequently used (72.7\%) by most students (86.8\%) and were used for self-assessment (75.0\%) and identification of deficient content (65.6\%). It was possible to develop a mobile application that partially met the needs reported by users. Conclusion: Applications are tools that assist in the study and self-assessment for selection of medical residency, however the available ones do not meet the user's needs. The prototype of a mobile application that aggregates questions was developed, adapted to the profile of this user, which supplies shortcomings pointed out, such as free access and the possibility of creating personalized tests.

Keywords: Internship and Residency. Mobile Applications. Medical Education. Training.

\section{Introdução}

O treinamento em uma área específica da medicina para a obtenção do título de especialista é um caminho natural dos médicos do Brasil. No ano de 2017 , a maioria (62,5\%) dos médicos no Brasil possuía, ao menos, um título de especialista e a maioria dos novos médicos $(80,2 \%)$ pretendia cursar residência médica ${ }^{1}$. A conclusão de um programa de residência médica ou a obtenção de título via Sociedade de Especialidade Médica são os caminhos oficiais para a conquista do título de especialista, sendo a primeira opção a mais frequente. 0 ingresso em um programa de residência se dá mediante processos seletivos de ampla concorrência, sendo que em 2017 foram ocupadas 16.499 vagas no primeiro ano de residência.

Cursos preparatórios para as provas de residência médica, cujo principal objetivo é treinar alunos para obter bons resultados nas provas, proliferam-se diante da demanda e da concorrência. Para isso, dispõem de materiais pedagógicos, como livros e apostilas, estruturados de acordo com as tendências das últimas provas. Em um contexto geral, os alunos estudam ou revisam matérias por meio de cursos extracurriculares e/ou utilizando materiais dos cursos preparatórios, incluindo ferramentas digitais². 
O uso de ferramentas digitais e plataformas móveis é altamente difundido no ambiente do acadêmico de medicina. Cerca de $85 \%$ dos alunos utilizam algum dispositivo de computação móvel para acesso à informação ${ }^{3}$. Essa popularização dos smartphones se deve à portabilidade, flexibilidade, acesso à multimídia e acesso rápido à informação, sem falar no custo mais baixos e comparado com computadores ${ }^{4,5}$.

Em um estudo envolvendo alunos do internato na Universidade de Brasília, mais da metade dos entrevistados $(53,7 \%)$ consideravam crucial a participação nos cursos preparatórios para a aprovação nas provas de residência médica. Porém, uma parcela desses alunos não frequentaria esses cursos por não ter condições financeiras ${ }^{6}$.

Uma pesquisa investigou estudantes, residentes e membros do corpo docente de faculdades de medicina canadenses e demonstrou o uso generalizado de smartphones, por todos os 1.210 entrevistados, para responder questões clínicas e encontrar informações médicas ${ }^{7}$.

Assim, percebe-se que a tecnologia móvel já foi incorporada na área da medicina, e a inclusão de tecnologias educacionais, como o uso de aplicativos, pode auxiliar no processo ensino-aprendizagem dos estudantes ${ }^{3,8}$. Contudo, não há estudo sistemático sobre a utilidade e opinião sobre o uso de aplicativos com conteúdo para residência médica e seu eventual efeito no desempenho do usuário nos processos de seleção. Frente a isso, o presente estudo propôs a construção de um aplicativo móvel de estudo para as provas de residência médica, baseado nas perspectivas e opiniões de usuários, estudantes de medicina, avaliados por um estudo analítico transversal.

\section{Métodos}

\section{Avaliação das perspectivas}

Na primeira etapa do projeto foi realizado um estudo analítico transversal através da aplicação de questionário on-line na plataforma Google Forms visando investigar o entendimento dos alunos sobre o processo de residência médica. Antes de responderem às questões, foi apresentado o Termo de Consentimento Livre e Esclarecido (TCLE) intitulado "Avaliação das perspectivas dos estudantes de medicina quanto à Residência Médica e seu processo de seleção", aprovado pelo Comitê de Ética e Pesquisa em Seres Humanos (CEP/SD) do Setor de Ciências da Saúde da Universidade Federal do Paraná (UFPR), com o parecer número 2.685.185 na data de 30 de maio de 2018. 
A amostra foi realizada por conveniência e contemplou estudantes do terceiro ao sexto ano de medicina da UFPR. O período de coleta dos dados foi entre agosto e outubro de 2018. Para aqueles que concordaram com o TCLE, foi disponibilizada uma enquete com 17 perguntas sobre seus dados demográficos, impressões pessoais sobre a residência médica e o perfil do uso de aplicativos móveis como ferramenta de estudo para as provas. Para análise estatística, os participantes foram divididos em dois grupos: grupo período clínico (estudantes do terceiro e quarto ano) e grupo internato (estudantes do quinto e sexto ano). As respostas foram analisadas utilizando o software GraphPad Prism 7. Para comparação das proporções entre os grupos foi utilizado o teste exato de Fisher, sendo considerado o valor de $p<0,05$ como critério de significância estatística.

\section{Desenvolvimento do aplicativo}

Foi realizado o levantamento e a análise de requisitos em reunião com a equipe do projeto para a definição das funcionalidades do aplicativo e da criação do sistema para gerenciamento do banco de provas. Foram avaliados aplicativos do mesmo seguimento em artigos científicos e na loja de programas móveis Play Store da Google. Não foram encontrados aplicativos semelhantes na base de dados do Sistema Online de Busca e Análise de Literatura Médica (MEDLINE - Medical Literature Analysisand Retrieval System Online).

$\mathrm{Na}$ busca na loja de aplicativos da Google, foram encontrados alguns modelos e avaliados conforme os seguintes parâmetros: preço, presença de anúncios, quantidade e atualidade das questões, divisão por áreas e/ou assuntos, salvamento do progresso, leiaute, usabilidade, interação dos usuários, necessidade de internet e outras observações. Alguns aplicativos eram pagos e não puderam ser examinados. Aplicativos gratuitos, em sua maioria: continham anúncios; tinham pequena base de questões e muitas desatualizadas; permitiam seleção de questões por área, mas nem todos salvavam o progresso de resolução; possuíam leiautes e usabilidade regulares e a maioria não permitia interação entre usuários. Alguns permitiam o uso somente on-line e apenas um dos aplicativos oferecia algumas questões com comentários de profissionais.

Com base nos aplicativos avaliados, foi realizado o desenho de arquitetura e detalhamento do novo aplicativo. Para a codificação foi utilizado o Android Studio como ambiente integrado de desenvolvimento (IDE - Integrated Development Environment), o SQLite Studio como gerenciador de banco de dados, e as linguagens de programação Java, 
XML e SQL. Para o desenvolvimento do sistema de gerenciamento foi utilizado o Netbeans como IDE, a linguagem de programação PHP e a interface Template ERP do framework Adianti Solutions. O sistema foi hospedado na plataforma Firebase Hosting. Como solução para armazenamento de informações foi utilizado o Firebase Firestore, para armazenamento do banco de questões e dados dos usuários, e a extensão Realm, para as preferências de uso. Outras ferramentas utilizadas foram o Firebase Authentication para autenticação de e-mails e o Adobe Photoshop CS6 para edição de imagens. Sucederam-se etapas de criação de funcionalidades, integração do sistema e do aplicativo com o banco de questões, configurações de usabilidade e testes de software.

\section{Resultados}

\section{Avaliação das perspectivas}

A amostra da primeira etapa foi composta de 127 alunos, sendo 85 do sexo feminino (66,92\%), com média de idade de 23,72 $\pm 3,04$ anos. Destes, 69 alunos (53,54\%) eram do ciclo clínico (terceiro e quarto ano) e 58 (46,46\%) do internato (quinto e sexto ano).

A maioria dos alunos $(93,7 \%, n=119)$, pretendia realizar prova de residência e $8(6,3 \%)$ ainda não haviam decidido. Não houve diferença estatística $(p=0,72)$ entre a pretensão de realizar a prova quando comparado o período clínico (92,75\%) e o internato (94,83\%).

As especialidades de escolha estão representadas no gráfico A da Figura 1(anexos). A maioria dos participantes $(69,3 \%)$ não acreditava que essa escolha seria a definitiva; quando comparado entre os grupos, não houve diferença estatística $(p=0,70)$. Sessenta e oito $(53,5 \%)$ participantes haviam pesquisado quais hospitais ofereciam residência na sua especialidade de interesse.

De todos os 127 estudantes da amostra, 68 sabiam quais conteúdos eram abordados na prova de residência médica. A diferença de conhecimento dos conteúdos abordados nas provas de residência médica entre os alunos do período clínico $(52,17 \%)$ comparados com os alunos do internato $(55,17 \%)$ não foi estatisticamente significativa $(p=0,85)$.

As regiões de escolha para realizar a prova de residência (sendo possível a opção por mais de uma região por aluno) estão representadas no gráfico B da Figura 1.

Foi perguntado aos participantes se eles sabiam o valor médio da inscrição para a prova de residência médica. Cerca de metade $(50,4 \%)$ acreditava que os preços estavam entre 300 e 500 reais; $22,8 \%$ entre 100 e 300 reais e outros $23,6 \%$ entre 500 e 1.000 reais. Três pessoas $(2,4 \%)$ pensavam que o preço iria até 100 reais e uma pessoa $(0,8 \%)$ achou 
que custava mais de mil reais. As perspectivas quanto ao valor médio anual de um curso preparatório estão sumarizadas no gráfico $\mathrm{C}$ da figura 1.

Dos 127 participantes, $38(29,92 \%)$ já haviam iniciado os estudos para a prova de residência. Dentre estes, 17 (24,64\%) eram do grupo clínico e 21 (36,21\%) do grupo internato, sem diferença estatística entre os grupos $(p=0,17)$. Os métodos de estudo para as provas de residência que utilizavam, em ordem de mais escolhido, foram: apostilas $(97,4 \%)$, resumos e provas antigas (68,4\% para cada), videoaulas $(63,2 \%)$ e livros $(39,5 \%)$. Dos respondentes ( $n=38), 86,8 \%$ (33) relataram utilizar aplicativos como meio de estudo.

Em relação ao uso de aplicativos como forma de estudo para as provas de residência médica ( $n=32$ respondentes), as repostas foram graduadas pela escala de Likert e estão sumarizadas na Figura 2 (anexos). A grande maioria concordava ou concordava plenamente com a afirmação de utilizar aplicativos com conteúdo para prova de residência com frequência (72,73\%) e com a afirmação de os mesmos aplicativos auxiliarem nos estudos (84,37\%). Cerca de $43,75 \%$ dos participantes não consideram os aplicativos motivadores para o estudo. A maioria concordava ou concordava plenamente que os aplicativos eram uma forma de autoavaliação (75\%) e que serviam para identificar conteúdos que não tinham entendido previamente $(65,64 \%)$. Cerca de $34,38 \%$ eram indiferentes quanto a afirmação de os aplicativos os levarem a buscar bibliografia complementar.

\section{Desenvolvimento do aplicativo}

O software para gerenciamento do banco de questões das provas de residência é um sistema web com espaços no qual se pode adicionar a questão, universidade, área de conhecimento, especialidade, referência de imagem e ano. Também é possível visualizar todas as questões, editá-las ou excluí-las, se necessário. Somente pessoas autorizadas têm acesso ao sistema e esse controle é feito por um processo de autenticação. Na Figura 3 (anexos) é apresentado uma captura de tela do sistema criado.

O nome escolhido para o aplicativo foi Quero Ser R1, uma alusão ao fato de os estudantes estarem buscando uma vaga no primeiro ano de residência. Em seu primeiro contato com o aplicativo, é apresentado a tela de login, que possui a logomarca e um botão que permite o usuário entrar com seu e-mail da Google; esse processo é necessário para vincular os dados de utilização com o usuário. Ainda no primeiro acesso, após efetuar a autenticação, é apresentado um tutorial sobre as funções principais do aplicativo. Após a leitura das instruções, o usuário é direcionado para a tela principal, na qual pode selecionar as funcionalidades da aplicação. 
No módulo Exercícios é possível criar e resolver um ou mais caderno(s) de questões filtrados por área de conhecimento, especialidade, ano e instituição. Ao final de cada questão respondida pode-se escrever um comentário público sobre aquela questão e ver todos os outros comentários escritos por outras pessoas, podendo marcá-los como "Gostei”/“Não gostei”, ou até denunciar algum comentário se assim achar pertinente. Os comentários denunciados serão avaliados pela equipe desenvolvedora e, se necessário, serão excluídos. Pode-se interromper a resolução e voltar a responder a partir da pergunta em que parou. Também é possível visualizar o histórico de todas as questões já respondidas. A diferença da função Simuladoé permitir que o usuário responda um caderno selecionando uma área de conhecimento, quantas questões deseja responder e o tempo para responder cada questão; se necessário, é possível parar e continuar o simulado, posteriormente.

As informações de acertos e erros de todos os cadernos de provas do módulo Exercícios são armazenadas e mostradas na tela Desempenho. Essas informações são divididas pelas áreas de clínica médica, clínica cirúrgica, ginecologia/obstetrícia, pediatria e medicina preventiva; também é possível visualizar a porcentagem de acertos em cada área. No módulo Ranking, os dados de cada usuário são colocados em uma lista ordenada pela quantidade de acertos; podendo ser listados por acertos gerais ou por acertos em cada área. Se desejar, o usuário pode não participar desse ranking. A opção Perfilé uma tela que irá apresentar informações sobre a utilização do software e os dados sobre a equipe que trabalha em seu desenvolvimento. A Figura 4 (anexos) mostra exemplo de telas do aplicativo.

\section{Discussão}

A maior parte dos alunos entrevistados tinha vontade de fazer a residência médica e apresentava conhecimento do valor das provas e dos cursos preparatórios, porém não havia começado os estudos para a prova de residência. Mais de $80 \%$ dos participantes do estudo afirmaram utilizar aplicativos como forma de estudo e que esses aplicativos auxiliariam no processo de aprendizagem, sendo importantes também como uma forma de autoavaliação e na identificação de conteúdo que não havia sido estudado anteriormente. Porém, quase metade da amostra não considerava os aplicativos disponíveis, no momento, atrativos para o estudo. Essa falta de interesse pelos aplicativos pode ser consequência de diversos fatores como, por exemplo, falta de gamificação (ludificação) e explicações sobre as 
questões. Assim, o aplicativo Quero ser $R 1$ foi desenvolvido com o objetivo de suprir essas lacunas relatadas pelos estudantes.

Conforme o previsto na Demografia Médica no Brasil 2018¹, a grande maioria dos estudantes de medicina da UFPR pretende fazer a prova de residência médica. Não houve diferença estatisticamente significativa na decisão de fazer ou não a prova, entre os alunos dos ciclos clínico e internato.

No contexto brasileiro, as áreas de escolha dos egressos para residência médica são: pediatria $(12,3 \%)$, clínica médica $(11,5 \%)$, cirurgia geral $(8,8 \%)$, ginecologia e obstetrícia $(8,6 \%)$ e anestesiologia (7,1\%); havendo preferência para as regiões do sudeste e sul do país ${ }^{1}$. As predileções dos egressos coincidem com a distribuição dos médicos com título de especialista ${ }^{1}$. Seguindo em parte a Demografia Médica, a amostra de estudo teve maior preferência, em ordem decrescente, pelas especialidades de clínica médica (19,7\%), cirurgia geral (17,3\%) e ginecologia e obstetrícia (8,7\%), e pretende realizar a prova na região sudeste $(61,4 \%)$ e sul $(45,7 \%)$ do país. Os dados não permitiram concluir que os alunos do internato estivessem mais decididos sobre qual especialidade seguir quando comparados a alunos no ciclo clínico.

Cerca de metade da amostra (53,5\%) chegou a pesquisar os hospitais que oferecem sua especialidade e a mesma porcentagem já sabia sobre os conteúdos que são abordados na prova, não havendo diferença entre os períodos comparados.

Em relação ao valor médio para realizar as provas de residência e dos cursos preparatórios, a maioria dos alunos tinha conhecimento acerca dos valores pesquisados para a região de Curitiba (PR). Cerca de metade dos alunos respondeu como preço médio de inscrição em prova de residência a faixa de 300 a 500 reais, o que coincide com pesquisa dos autores sobre o valor médio de oito comissões do Paraná que oferecem o serviço de residência médica (Complexo Hospital de Clínicas, Hospital do Trabalhador, Hospital Pequeno Príncipe, Hospital Santa Casa de Curitiba, Instituto de Neurologia e Cardiologia de Curitiba, Secretaria Municipal de Saúde de Curitiba, Hospital Cruz Vermelha e Hospital Universitário Cajuru/Hospital e Maternidade Santa Brígida). Sobre o que a amostra considerava ser o preço anual médio de um curso preparatório, a maioria $(59,1 \%)$ escolheu a faixa de 10.000 a 15.000 reais por ano, o que também coincide com a pesquisa dos autores sobre o preço de sete cursos preparatórios de um e de dois anos ofertados na região do Paraná, com valor ao redor de 10.500 reais anuais. Apesar do perfil da maioria dos recém- 
graduados em medicina no Brasil estar na faixa de renda elevada ${ }^{1}$, o alto preço dos cursos preparatórios é fator limitante para alguns alunos ${ }^{2}$.

Em proporção, mais alunos do internato haviam começado seus estudos para a prova de residência, porém essa diferença não foi estatisticamente significante. Podemos inferir que, nessa amostra, alunos que estão se preparando para a residência fazem isso desde o ciclo clínico. Daqueles que começaram, a maioria estuda por apostilas, resumos e provas antigas, e a minoria por livros; uma hipótese para isso é que livros costumam ser um método lento de estudo e as apostilas e os resumos são mais práticos e objetivos ${ }^{6}$.

A maioria dos estudantes usava aplicativos com frequência, como ferramenta de preparação para as provas de residência, que os auxiliavam a identificar conteúdos prévios não consolidados e a se autoavaliarem. Os aplicativos não pareceram motivadores, pois talvez faltem elementos de gamificação nesses aplicativos. A amostra de estudo pareceu indiferente quanto aos aplicativos os levarem a procurar bibliografia complementar; uma hipótese, segundo relatos lidos nos comentários dos aplicativos na loja virtual, é que os alunos desejam que ao término de cada questão respondida apareça explicações sobre as assertivas estarem certas ou erradas.

Sendo assim, o aplicativo Quero ser $R 1$ foi desenvolvido buscando suprir as necessidades relatadas pelos alunos nos questionários. 0 sistema web para gestão do banco de questões é totalmente eficaz, uma vez que é bastante simples acrescentar um novo exercício e editar e/ou excluir os já adicionados. As questões são armazenadas numa base de dados própria do projeto, sendo possível traçar uma plotagem das informações de todos os usuários e listá-los em um ranking, como forma de proporcionar competitividade entre os utilizadores.

O aplicativo desenvolvido é gratuito e conta, em sua fase inicial, com um pequeno banco de questões de provas do último ano. Por ser de acesso livre, pode servir de valiosa ferramenta para aqueles que não podem pagar um curso preparatório ${ }^{2,6}$.

Atendendo uma necessidade encontrada nos comentários de outros aplicativos, 0 aplicativo permite criar cadernos de provas, dividindo por áreas de conhecimento e especialidades, e salvar o progresso de cada caderno permitindo prosseguimento posterior. Possui leiaute simples, intuitivo e de fácil usabilidade. É necessário estar on-line para o uso do aplicativo, pois o banco de provas está na nuvem; assim, o aplicativo não sobrecarrega a memória do celular e é possível a atualização constante e simultânea das questões. Ao permitir comentários dos usuários em cada questão respondida é possível o 
compartilhamento de conhecimento e informações, seguindo os conceitos da web $2.0^{9} \mathrm{e}$ atendendo o anseio dos usuários em ter explicações sobre o conceito correto das respostas. A função Desempenho ajuda na autoavaliação e a função Ranking é um elemento de gamificação.

O estudo apresentou algumas limitações, como o recrutamento de voluntários em um único centro, o que pode impactar dados como o de opções regionais de escolha das provas a realizar e o desenvolvimento do aplicativo ainda restrito à plataforma Android.

\section{Conclusão}

Aplicativos são ferramentas que auxiliam no estudo e na autoavaliação para seleção de residência médica. São utilizados com frequência por acadêmicos de medicina para este fim, porém os atualmente disponíveis não suprem as necessidades do usuário, na opinião da amostra investigada. Foi desenvolvido com sucesso o protótipo de um aplicativo móvel agregador de questões, adequado ao perfil deste usuário, que supre carências apontadas, como acesso grátis e possibilidade de criação de testes personalizados.

Estudos subsequentes devem focar na análise da efetividade de ferramentas digitais, especialmente aplicativos, no aprendizado e sucesso dos acadêmicos de medicina nos testes seletivos de residência médica.

\section{Agradecimentos}

Agradecimento ao Conselho Nacional de Desenvolvimento Científico e Tecnológico (CNPq), que financiou o projeto pelo Programa Institucional de Bolsas de Iniciação em Desenvolvimento Tecnológico e Inovação - PIBITI (Gabriel Souza) e Bolsa de Produtividade em Desenvolvimento Tecnológico e Extensão Inovadora - PD (Fernando Dias). Ao financiamento parcial obtido pelo edital PPSUS Edição 2015-Fundação Araucária-PR/SESAPR/CNPq/MS-Decit. Agradecimento às alunas Alana Gomes Pecorari e Jaqueline Alves Zwierzikowski, que participaram da coleta de dados e, em especial, ao aluno Alisson Lopes de Sousa Freitas, pela ajuda na programação. Agradecimento aos profissionais de Letras Nelson Luiz Garcia de Oliveira e Tereza Mariko Yamashita que fizeram a correção gramatical. 


\section{Referências}

1. Scheffer M, Cassenote A, Guilloux AGA, Biancarelli A, Miotto BA, Mainardi GM. Demografia Médica no Brasil 2018 [Internet]. São Paulo: SP: FMUSP, CFM, Cremesp; 2018 [citado 30 de setembro de 2019]. 286 p. Disponível em: http://www.flip3d.com.br/web/pub/cfm/index10/?numero=15\&edicao=4278

2. Chehuen Neto JA, Sirimarco MT, Choi CMK, Fava AS, Oliveira L de RS, Cunha PHM da. Cursinhos preparatórios para prova de residência médica: expectativas e opiniões. RevBrasEduc Médica. junho de 2009;33(2):205-10.

3. Oliveira EY, Crosewski NI, Silva ALM, Ribeiro CTD, de Oliveira CM, Fogaça RTH, et al. Profile of Educational Technology Use by Medical Students and Evaluation of a New Mobile Application Designed for the Study of Human Physiology. J MedSyst [Internet]. outubro de 2019 [citado 19 de novembro de 2019];43(10). Disponívelem: http://link.springer.com/10.1007/s10916-019-1438-7

4. Wallace S, Clark M, White J. 'It's on my iPhone': attitudes to the use of mobile computing devices in medical education, a mixed-methods study. BMJ Open [Internet]. $1^{\circ}$ de janeiro de 2012 [citado $1^{\circ}$ de outubro de 2019];2(4). Disponívelem: https://bmjopen.bmj.com/content/2/4/e001099

5. Lumsden CJ, Byrne-Davis LMT, Mooney JS, Sandars J. Using mobile devices for teaching and learning in clinical medicine. ArchDisChild - EducPract. $1^{\circ}$ de outubro de 2015;100(5):244-51.

6. Silva SM e, Rosa VF, Brandão PR de P, Oliveira AC de, Oliveira PG de, Sousa JB de. Cursos preparatórios para a residência médica: visão dos estudantes de medicina. Rev Colégio Bras Cir. outubro de 2011;38(5):349-54.

7. Boruff JT, Storie D. Mobile devices in medicine: a survey of how medical students, residents, and faculty use smartphones and other mobile devices to find information. J Med LibrAssoc JMLA. janeiro de 2014;102(1):22-30.

8. Briz-Ponce L, Juanes-Méndez JA, García-Peñalvo FJ, Pereira A. Effects of Mobile Learning in Medical Education: A Counterfactual Evaluation. J Med Syst. junho de 2016;40(6):136.

9. O'Reilly T. What Is Web 2.0: Design Patterns and Business Models for the Next Generation of Software [Internet]. 2007 [citado 6 de outubro de 2019]. Disponível em: https://mpra.ub.uni-muenchen.de/4580/ 


\section{Anexos}

Figura 1: Perspectivas de escolha de especialidades, regiões para realização das provas e preço médio dos exames.
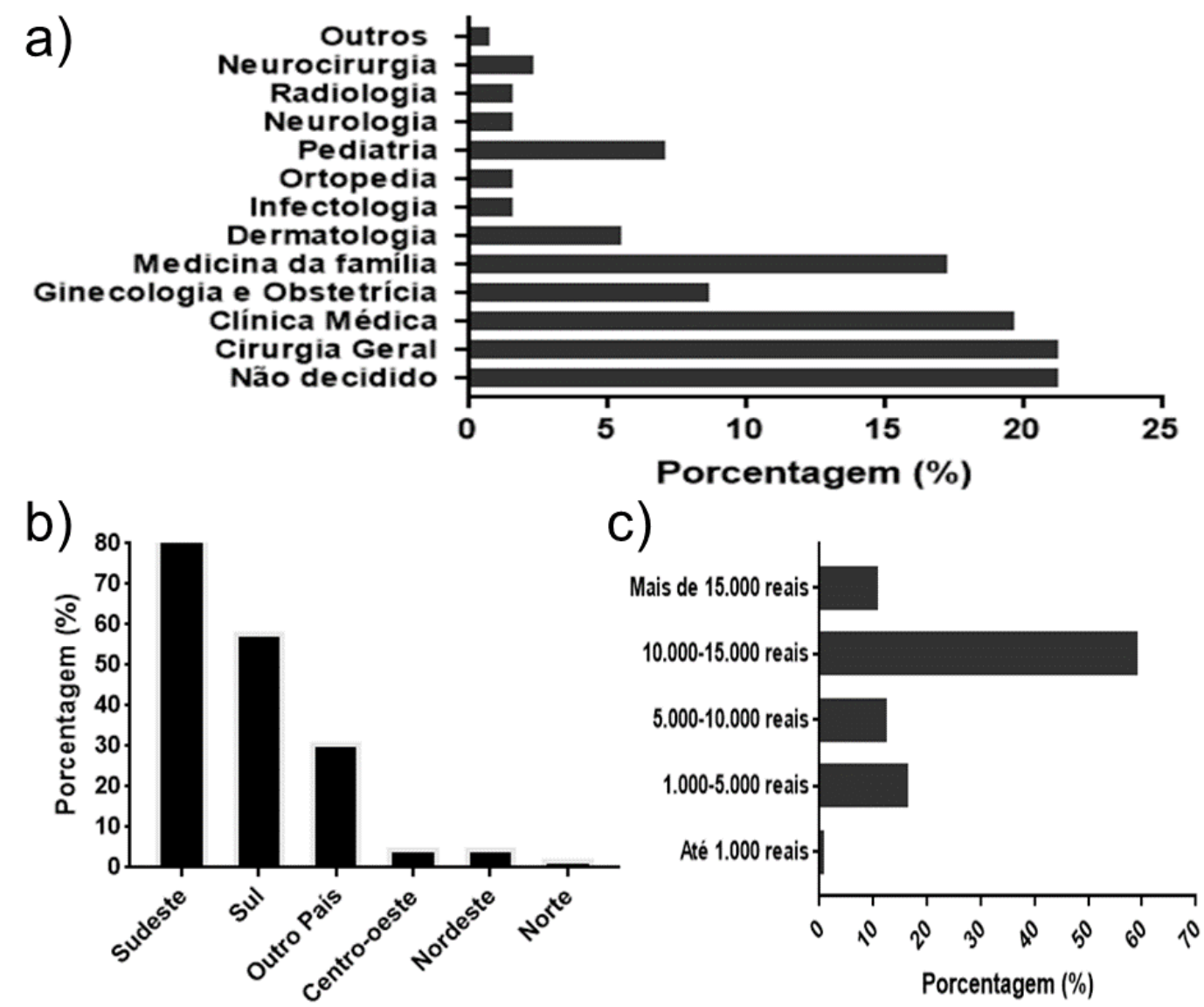

Legenda: a) especialidades pretendidas; $b$ ) regiões de escolha; $c$ ) perspectivas de preço anual de um curso preparatório

Fonte: os autores 
Figura 2: Opinião dos alunos quanto ao uso de aplicativos de estudo para provas de residência médica

a) "Utilizo os aplicativos com conteúdo para prova de residência médica com frequência em meu dia-a-dia" ( $n=33$ )

b) "Os aplicativos com conteúdo para prova de residência médica me auxiliaram no estudo para a prova de residência médican $(n=32)$

c) "Fiquei motivado a estudar em consequência do uso de aplicativos com conteúdo para prova de residência médica" $(n=32)$

d) "Usei aplicativos com conteúdo para prova de residência médica como forma de me auto avaliar" $(n=32)$

e) "Os aplicativos com conteúdo para prova de residência médica me auxiliam a identificar os conteúdos que não tinha entendido previamente" $(n=32)$

f) "Os aplicativos com conteúdo para prova de residência médica me levaram a buscar bibliografia complementar" $(n=32)$

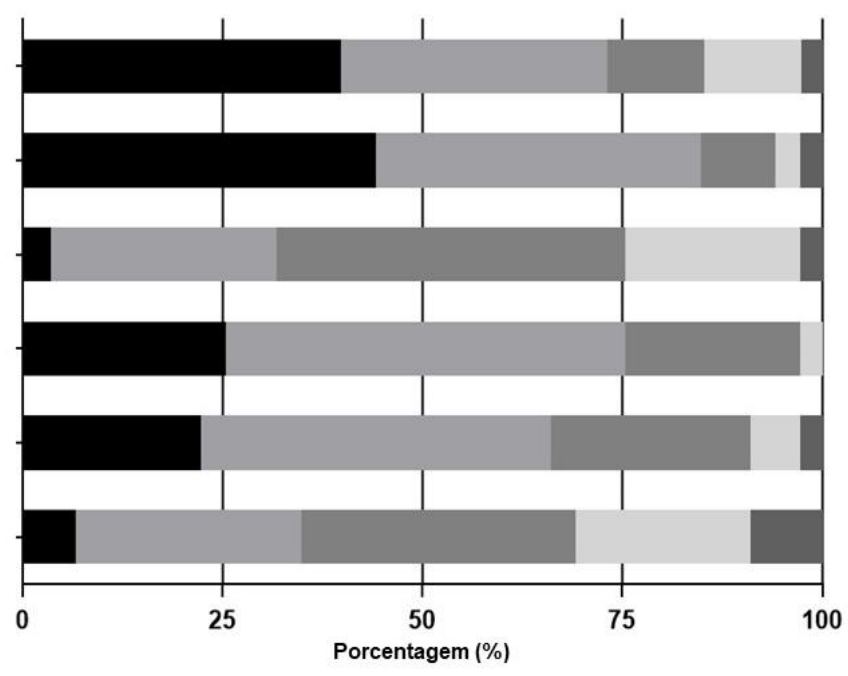

Concordo plenamente Concordo Sou indiferente Discordo Discordo plenamente

Fonte: os autores

Figura 3: Tela do sistema de gerenciamento do banco de questões

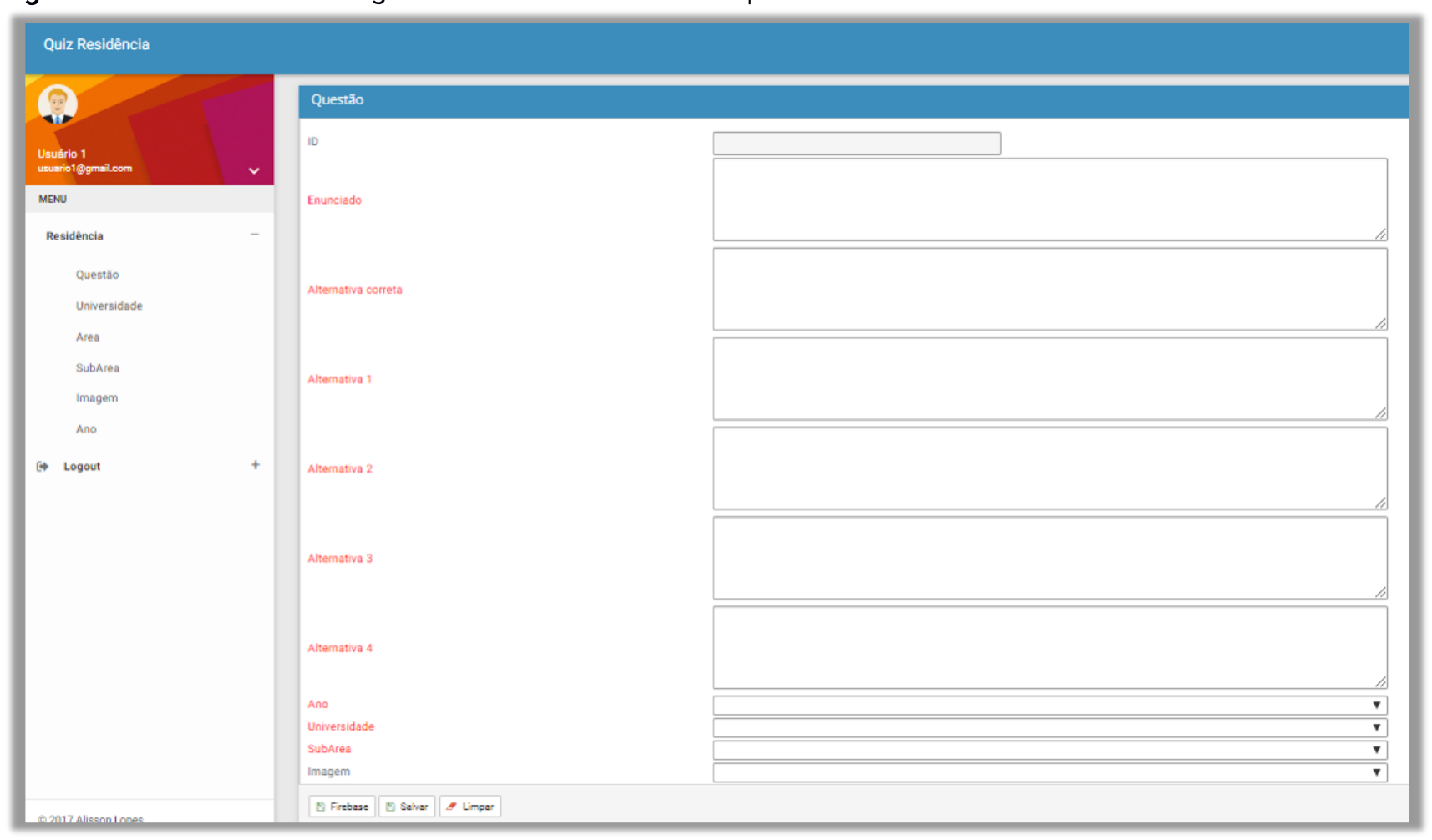

Fonte: os autores 
Figura 4: Telas do aplicativo Quero Ser R1

a)

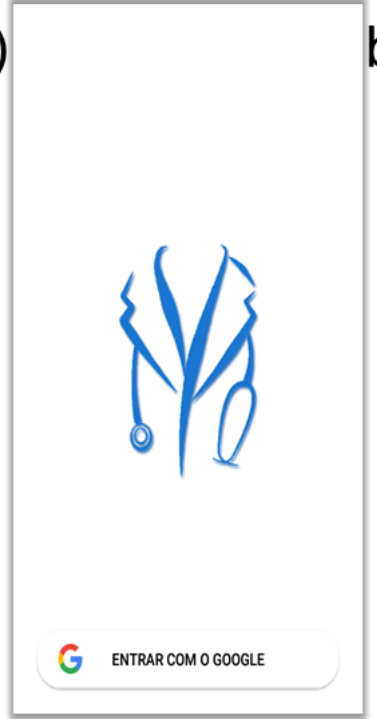

e)

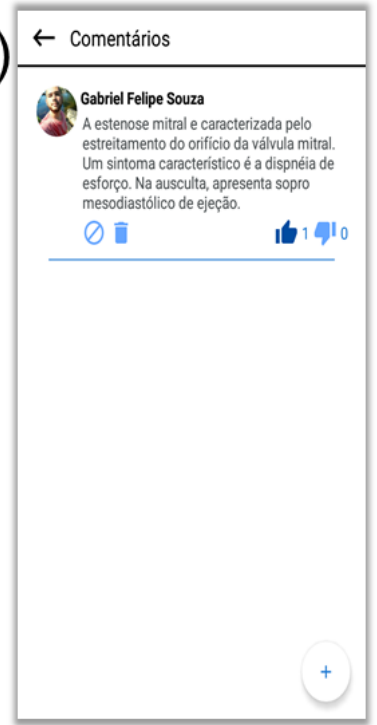

b)

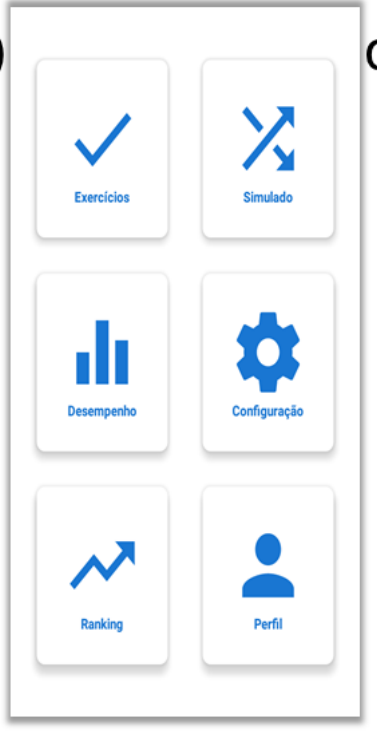

f)

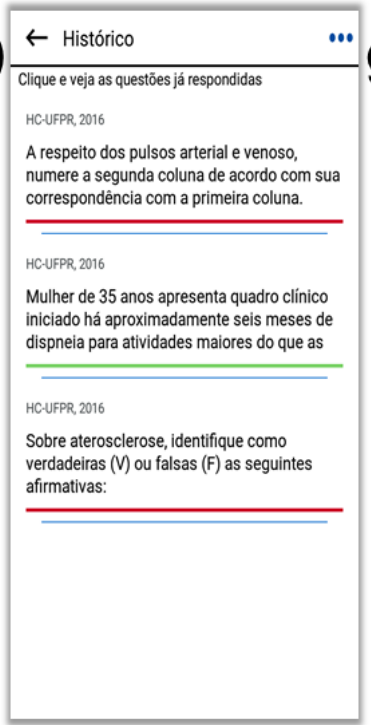

c)

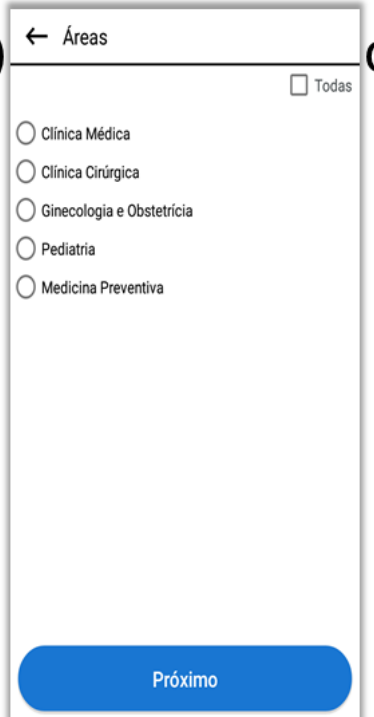

d

$\leftarrow$

HC.UPPR, 2016

Mulher de 35 anos apresenta quadro clínico iniciado há aproximadamente seis meses de cispneia para atividades maiores do que as faz uso de medicamento algum. No exame fisico, apresenta-se afebril, com pressão arterial de 120/80 UT, frequência cardíaca de 92 batimentos por minuto, ritmica, frequência respiratória de 16 respiraçōes por minuto. $\mathrm{Na}$ ausculta da região precordial, observou-se sopro na porção média da diástole. A doenç valvar dessa senhora é:

Dilatação da artéria pulmonar.

Estenose aórtica supravalvar.

Insuficiência mitral.

\section{Estenose mitral.}

Insuficiência aórtica.

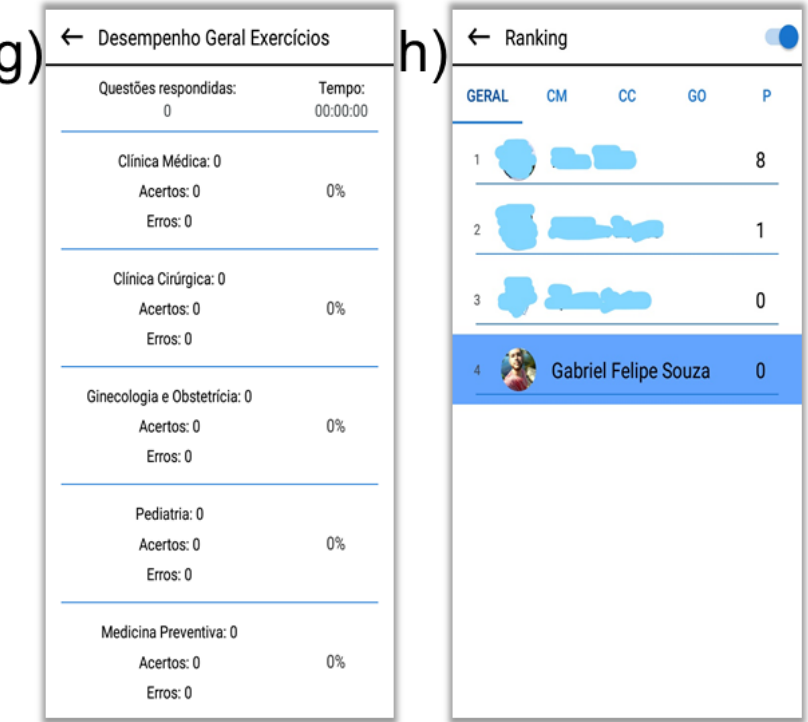

Legenda: a) tela de login; b) tela principal; c) seleção de áreas; d) tela de questão; e) comentários; f) tela de histórico; g) desempenho; h) ranking

Fonte: os autores 